\title{
Role of extracellular calcium and mitochondrial oxygen species in psychosine-induced oligodendrocyte cell death
}

\author{
V Voccoli ${ }^{1}$, I Tonazzini ${ }^{1}$, G Signore ${ }^{2}$, M Caleo ${ }^{3}$ and M Cecchini, ${ }^{*}$,
}

Globoid cell leukodystrophy (GLD) is a metabolic disease caused by mutations in the galactocerebrosidase (GALC) gene. GALC is a lysosomal enzyme whose function is to degrade galacto-lipids, including galactosyl-ceramide and galactosylsphingosine (psychosine, PSY). GALC loss of function causes progressive intracellular accumulation of PSY. It is widely held that PSY is the main trigger for the degeneration of myelinating cells and progressive white-matter loss. However, still little is known about the molecular mechanisms by which PSY imparts toxicity. Here, we address the role of calcium dynamics during PSY-induced cell death. Using the human oligodendrocyte cell line MO3.13, we report that cell death by PSY is accompanied by robust cytosolic and mitochondrial calcium $\left(\mathrm{Ca}^{2+}\right)$ elevations, and by mitochondrial reactive oxygen species (ROS) production. Importantly, we demonstrate that the reduction of extracellular calcium content by the chelating agent ethylenediaminetetraacetic acid can decrease intra-mitochondrial ROS production and enhance cell viability. Antioxidant administration also reduces mitochondrial ROS production and cell loss, but this treatment does not synergize with $\mathrm{Ca}^{2+}$ chelation. Our results disclose novel intracellular pathways involved in PSY-induced death that may be exploited for therapeutic purposes to delay GLD onset and/or slow down its progression.

Cell Death and Disease (2014) 5, e1529; doi:10.1038/cddis.2014.483; published online 20 November 2014

Globoid cell leukodystrophy (GLD), also known as Krabbe disease, is a childhood leukodystrophy triggered by mutations in the galactocerebrosidase (GALC) gene; the physiopathological hallmarks of GLD are progressive demyelination, reactive astrocytosis and microgliosis. ${ }^{1}$ GALC is a lysosomal enzyme essential for the normal catabolism of galacto-lipids, including galactosyl-ceramide and galactosyl-sphingosine (psychosine, PSY). GALC loss of function causes progressive accumulation of PSY, a cytotoxic metabolite that has been assumed as the main cause for GLD pathogenesis. ${ }^{2}$ PSY leads to Schwann cell and oligodendrocyte death, but still little is known about the molecular mechanisms by which PSY imparts toxicity. It has been demonstrated that PSY accumulates in cell membrane raft micro-domains, disrupting their architecture $^{3}$ and inhibiting protein kinase $C$ translocation to the plasma membrane. ${ }^{4}$ Recently, increased raft clustering was also reported in cultured dorsal root ganglion neurons prepared from the GLD murine model (i.e., the Twitcher mouse), and this was associated with the dysregulation of tyrosine kinase receptor $A$ membrane recruitment and ligandtyrosine kinase receptor $A$ activated endocytosis. ${ }^{5}$ PSY induces p53-mediated apoptotic cell death, ${ }^{6}$ tumor necrosis factor-related apoptosis, ${ }^{6,7}$ activation of secretory phospholipase $A 2,{ }^{8}$ cytochrome $C$ release from mitochondria and apoptosis activation via the caspase- 9 pathway. ${ }^{9}$ Moreover, several authors found that peroxisomal $\beta$-oxidation was significantly inhibited and very long-chain fatty acid levels and reactive oxygen species (ROS) production were increased in PSY-treated cells. ${ }^{10,11}$

Calcium $\left(\mathrm{Ca}^{2+}\right)$ is an essential ion for cell life, acting as a key second messenger in almost all cellular functions. It is well established that $\mathrm{Ca}^{2+}$ is one of the main second messengers involved in apoptotic cell death in neurons and in other cell types; sustained cytosolic $\mathrm{Ca}^{2+}$ increase can activate apoptosis. ${ }^{12}$ This can originate from extracellular influx or by release from intracellular stores like the endoplasmic reticulum. ${ }^{13}$ Importantly, mitochondria are also involved in $\mathrm{Ca}^{2+}$ homeostasis. ${ }^{14}$ Mitochondrial $\mathrm{Ca}^{2+}$ in basal conditions is maintained at low concentrations, but mitochondria are organelles that can take up high $\mathrm{Ca}^{2+}$ concentrations; indeed, different stimuli, such as nutrients, hormones or neurotransmitters that increase the cytoplasmic $\mathrm{Ca}^{2+}$ content also induce intra-mitochondrial $\mathrm{Ca}^{2+}$ increase. ${ }^{15}$ If this increase is relevant, ROS production increases and this is associated with mitochondrial membrane destruction, release of cytochrome $\mathrm{C}$ and apoptosis induction. ${ }^{16}$ During this process, proapoptotic $\mathrm{Bcl} 2$ family of proteins plays a crucial role by regulating the intracellular/mitochondrial $\mathrm{Ca}^{2+}$ content, and by inducing mitochondrial permeabilization, the essential step for cytochrome $\mathrm{C}$ release and caspase activation. ${ }^{12,17}$

${ }^{1}$ NEST, Istituto Nanoscienze-CNR and Scuola Normale Superiore, Piazza San Silvestro 12, 56127 Pisa, Italy; ${ }^{2}$ Center for Nanotechnology Innovation@ NEST, Istituto Italiano di Tecnologia, Pisa, Italy and ${ }^{3} \mathrm{CNR}$ Neuroscience Institute, via G. Moruzzi 1, 56124 Pisa, Italy

${ }^{*}$ Corresponding author: M Cecchini, NEST, Istituto Nanoscienze-CNR and Scuola Normale Superiore, Piazza San Silvestro 12, Pisa 56127 Italy; Tel: +39 050509459 ; Fax: +39 050509 417; E-mail: marco.cecchini@nano.cnr.it

Abbreviations: EDTA, ethylenediaminetetraacetic acid; $\mathrm{Ca}^{2+}$, calcium; DMSO, dimethyl sulfoxide; DMEM, Dulbecco's Modified Eagle Medium; GALC, galactocerebrosidase; GLD, Globoid cell leukodystrophy; NAC, N-acetyl-cysteine; PI, propidium iodide; PSY, psychosine; ROS, reactive oxygen species; PLA2, secretory phospholipase A2; S1P, sphingosine 1-phosphate; TMRM, tetra-metyl-rhodamine

Received 19.6.14; revised 22.9.14; accepted 06.10.14; Edited by A Finazzi-Agrò 
It has been reported that some sphingolipid metabolites, such as ceramides and sphingosine, can play a crucial role in many steps of apoptosis induction as regulators of some Bcl2 family proteins, by increasing intracellular $\mathrm{Ca}^{2+}$ levels and inducing mitochondrial stress. ${ }^{18}$ However, these mechanisms have never been explored during PSYinduced cell death.

In this article, we report on the role of intracellular $\mathrm{Ca}^{2+}$ dynamics during PSY-induced cell death in vitro. Using the human oligodendrocyte cell line MO3.13 and fluorescent probes, we measured $\mathrm{Ca}^{2+}$ variations in cytoplasm and mitochondria upon PSY administration until cell death. Moreover, we studied oxidative stress production in mitochondria by flow cytometry and time-lapse confocal fluorescence microscopy. Finally, in order to rescue cell viability in presence of PSY, we investigated the use of $\mathrm{Ca}^{2+}$ chelation in the extracellular medium, and its possible synergic effect with antioxidant treatment.

\section{Results}

PSY induces apoptotic and necrotic cell death. In order to evaluate PSY effects on cell viability, MO3.13 cells were treated for $24 \mathrm{~h}$ with different concentrations of PSY (1$10 \mu \mathrm{M})$ in serum-free condition to exclude the sphingolipid content present in serum (as sphingosine 1-phosphate $(\mathrm{S} 1 \mathrm{P})),{ }^{19}$ which could have hindered the PSY effect. After treatment, cells were harvested and stained with Annexin V-FITC conjugate and propidium iodide (PI), and analyzed by flow cytometry (Figure 1a).

Although only slightly reducing cell viability (Supplementary Figure S1a), serum starvation led to MO3.13 cell differentiation toward a more mature oligodendrocyte phenotype, as revealed by the marked change in cell morphology and the enhanced expression of myelin basic protein (Supplementary Figure S1b). As expected, PSYadministration induced a dosedependent cell death that started to be statistically significant

a
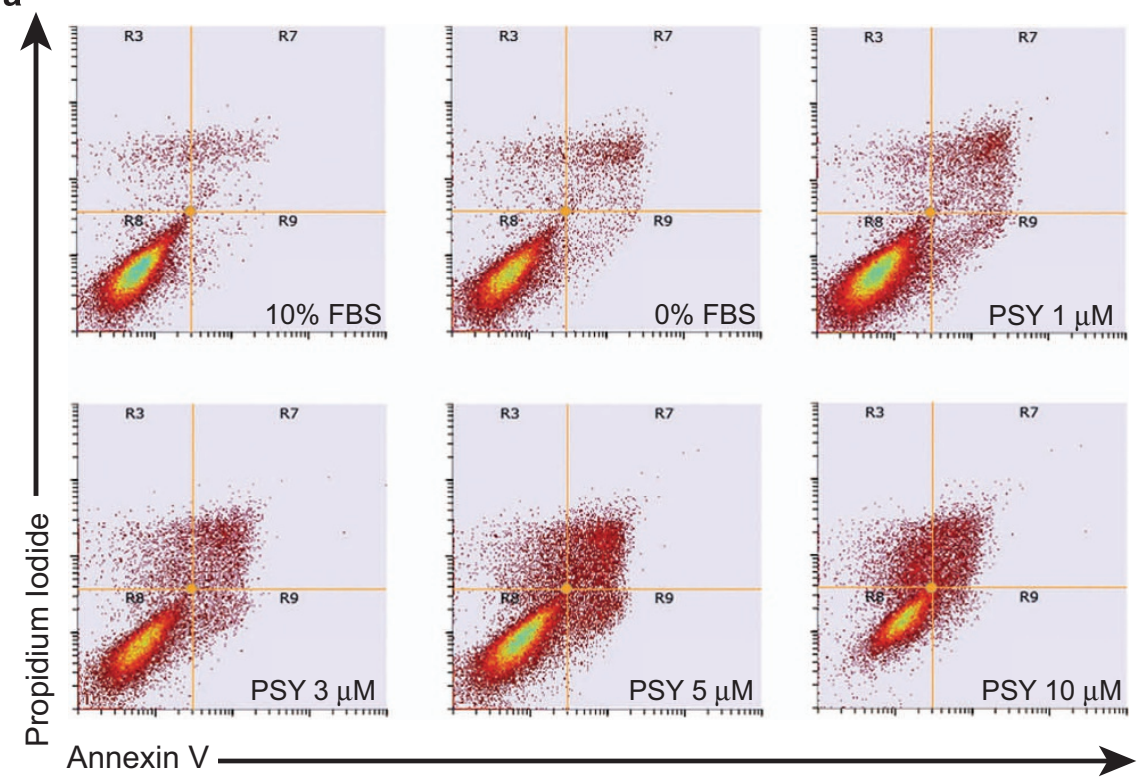

b
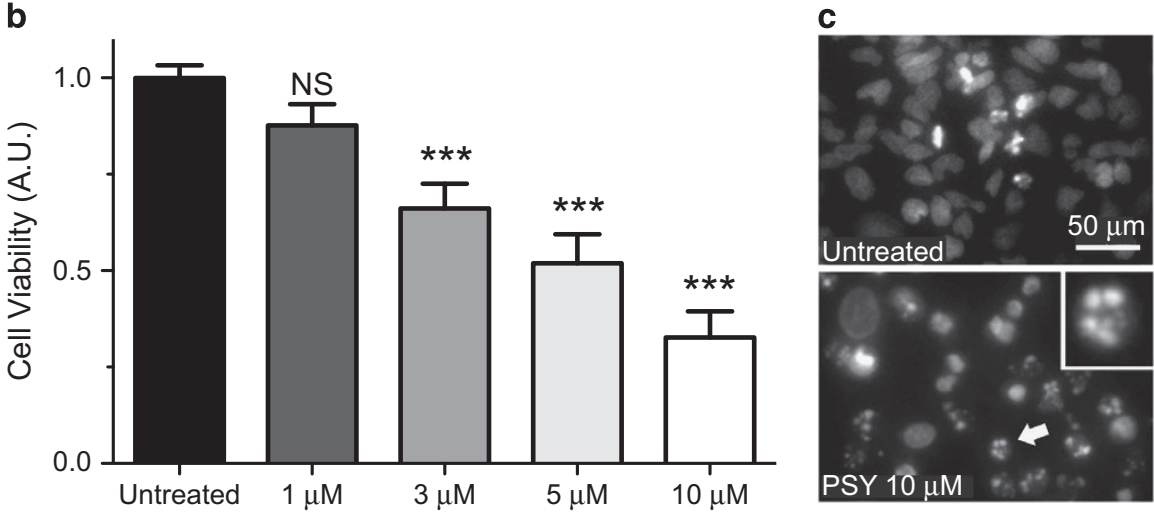

Figure 1 PSY induces dose-dependent cell death in MO3.13 cell line. (a) Representative dot-plots of MO3.13 cells stained with Annexin V/propidium iodide and analyzed by flow cytometry: R8, R9, R7 and R3 quadrants define the healthy, apoptotic, secondary necrotic and necrotic populations, respectively. (b) PSY dose-response quantification of cell viability ( $n=7$, one-way ANOVA (Dunnett's post-hoc test) PSY 1-10 $\mu \mathrm{M}$ versus Untreated). (c) Representative nuclear morphologies (imaged by Hoechst staining and fluorescence microscopy) for Untreated (top panel) and PSY-10- $\mu \mathrm{M}$-treated (bottom panel) cells. The bottom panel inset shows a zoomed image of the nucleus indicated by the white arrow. The presence of condensed/fragmented nuclei is a typical feature of apoptosis induction 
from the concentration of $3 \mu \mathrm{M}$. Specifically, cell viability was reduced by $34 \pm 6 \%(P<0.001), 48 \pm 7 \% \quad(P<0.001)$ and $67 \pm 7 \%(P<0.001)$ for $3 \mu \mathrm{M}, 5 \mu \mathrm{M}$ and $10 \mu \mathrm{M}$ PSY concentration, respectively (Figure $1 \mathrm{~b}$ ). Cell death occurred essentially by apoptosis, as demonstrated by the high percentage of Annexin V-positive cells and by the significant presence of condensed and fragmented nuclear morphologies (Figure 1c). The presence of a minor population of cells positive only for $\mathrm{PI}$ (Figure 1a, R3 quadrants) indicates that PSY also induced necrotic cell death.

PSY causes cytoplasmic and mitochondrial $\mathrm{Ca}^{2+}$ increases. Because $\mathrm{Ca}^{2+}$ is well recognized to be an important messenger during apoptosis, we measured its dynamics upon PSY administration by fluorescence confocal microscopy in living MO3.13 cells. The cells were co-stained with the calcium dye Fluo-3 AM and with the mitochondrial potential dye tetra-metyl rhodamine (TMRM) to also monitor mitochondrial potential loss during the last phases of apoptosis. $^{20}$ The stained cells were treated for $6-8 \mathrm{~h}$ with PSY $10 \mu \mathrm{M}$ and imaged by time-lapse confocal microscopy to follow the cytoplasmic $\mathrm{Ca}^{2+}$ kinetics from PSY administration $(t=0)$ until cell death occurred.

In the majority of the cells, PSY induced a first rapid $\mathrm{Ca}^{2+}$ peak after treatment ( $\approx 5-8 \mathrm{~min}$ ), followed by a second, slower $\mathrm{Ca}^{2+}$ increase that preceded cell death (Figure 2a). PSY acted also on mitochondrial potential causing a hyperpolarization followed by a progressive potential leak that ran out with TMRM loss of fluorescence (Figure 2a). During this phase, cells showed an evident membrane blebbing, typical hallmark of apoptosis execution. Among the cells that died by apoptosis, the lifetime distribution within the time-lapse interval ( $t \leq 8 \mathrm{~h}$ from PSY administration) showed two peaks (Supplementary Figure S2), suggesting the presence of two cell sub-populations with different tolerance to PSY. In particular, a group of cells had an average survival time of $35 \pm 2 \mathrm{~min}$, whereas the other one, more resistant to PSY, died at $t=165 \pm 11 \mathrm{~min}$ after treatment. Both these populations were characterized by a protracted phase of calcium dysregulation (Figure 2a). Differently, a minor population of MO3.13 cells reacted to PSY with a very fast $\mathrm{Ca}^{2+}$ increase coupled to mitochondrial potential loss, followed by cell lysis without membrane blebbing, indicating necrotic cell death induction (Figure $2 b$ ). These cells were extremely sensitive to PSY and died soon after treatment with an immediate calcium dysregulation. ${ }^{21}$ Control experiments were performed by treating cells with the same quantity of solvent (DMSO) and the cells failed to show any significant change in calcium or mitochondrial potential with respect to the baseline, demonstrating the specificity of PSY effects (Supplementary Figure S3a). Moreover, in order to demonstrate that TMRM fluorescence was correctly correlated to mitochondrial potential in our experimental set-up, we performed control experiments where TMRM signal variations were measured upon uncoupler (carbonyl cyanide m-chlorophenyl hydrazine, $25 \mu \mathrm{M}$ ) treatment (Supplementary Figure S8). This measure confirmed fluorescence-relative variations of the same order as those reported in Figures 2 and 3 .

In order to evaluate whether mitochondria could be involved in the observed cytoplasmic $\mathrm{Ca}^{2+}$ increase following PSY
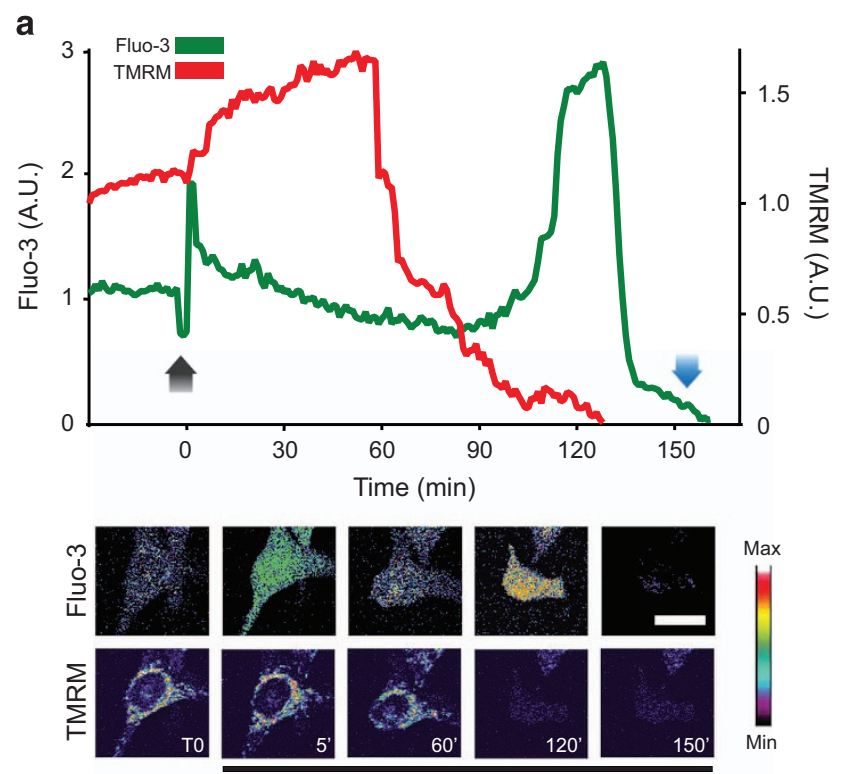

Time after PSY treatment

b
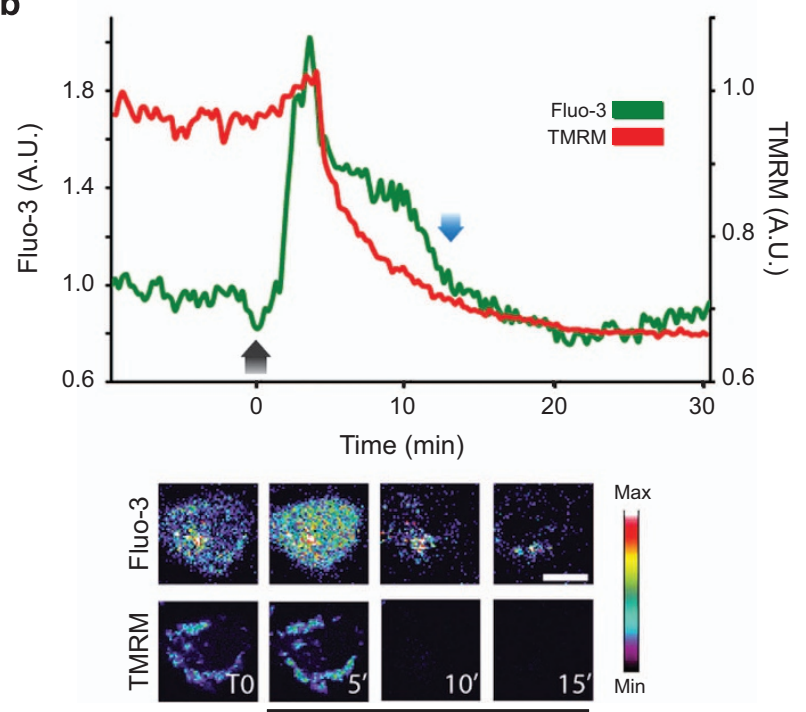

Time after PSY treatment

Figure 2 PSY induces cytoplasmic $\mathrm{Ca}^{2+}$ increases and mitochondrial depolarization. Representative traces of cytoplasmic $\mathrm{Ca}^{2+}$ (green lines) and mitochondrial potential (red lines) kinetics upon PSY $10 \mu \mathrm{M}$ administration ( $t=0$; black arrows) ( $n=3,>25$ analyzed cells). Fluo-3 and time-lapse fluorescence imaging of representative cells are reported below the traces. (a) Apoptotic $\mathrm{Ca}^{2+}$ dysregulation: PSY induces a first $\mathrm{Ca}^{2+}$ peak $(t=5-8 \mathrm{~min})$, and a second, more protracted, $\mathrm{Ca}^{2+}$ elevation before cell lysis (blue arrow). The mitochondrial potential shows a first hyper-polarization followed by progressive leak until cell lysis. (b) Necrotic $\mathrm{Ca}^{2+}$ dysregulation: only the first $\mathrm{Ca}^{2+}$ peak is present, coupled with fast mitochondrial depolarization until cell lysis that, in this case, occurred at $t=13 \mathrm{~min}$

treatment, MO3.13 cells were transfected with the MTCD2CPV mitochondrial $\mathrm{Ca}^{2+}$ FRET probe (see section 'Transfections' for details). We first verified the correct probe localization inside mitochondria: to this end, MTCD2CPVtransfected cells were stained with TMRM and analyzed by high-resolution confocal microscopy. High-magnification images were acquired along the $z$ axis by sequential acquisitions of the FRET signal and the TMRM fluorescence 
a

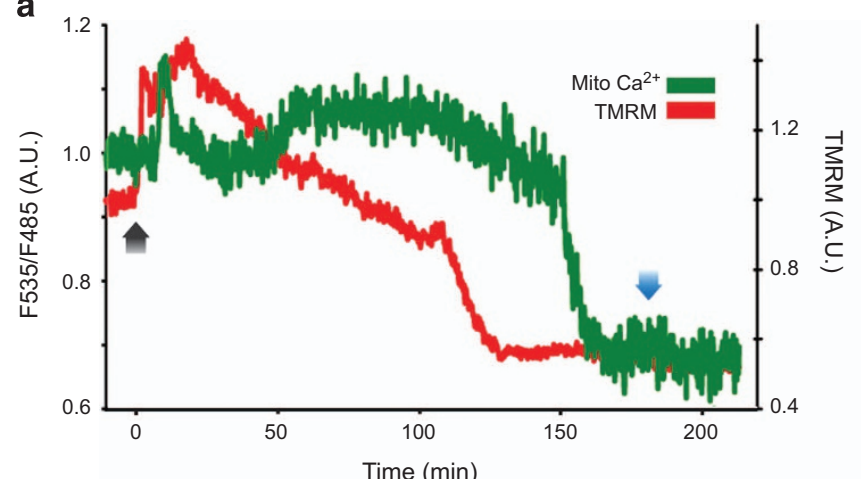

Time (min)

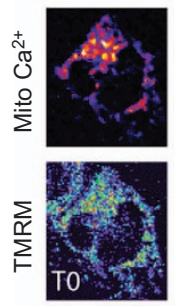

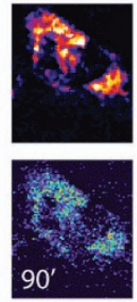

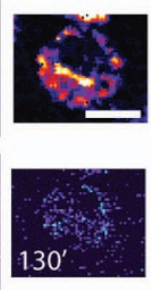

Time after PSY treatment

b
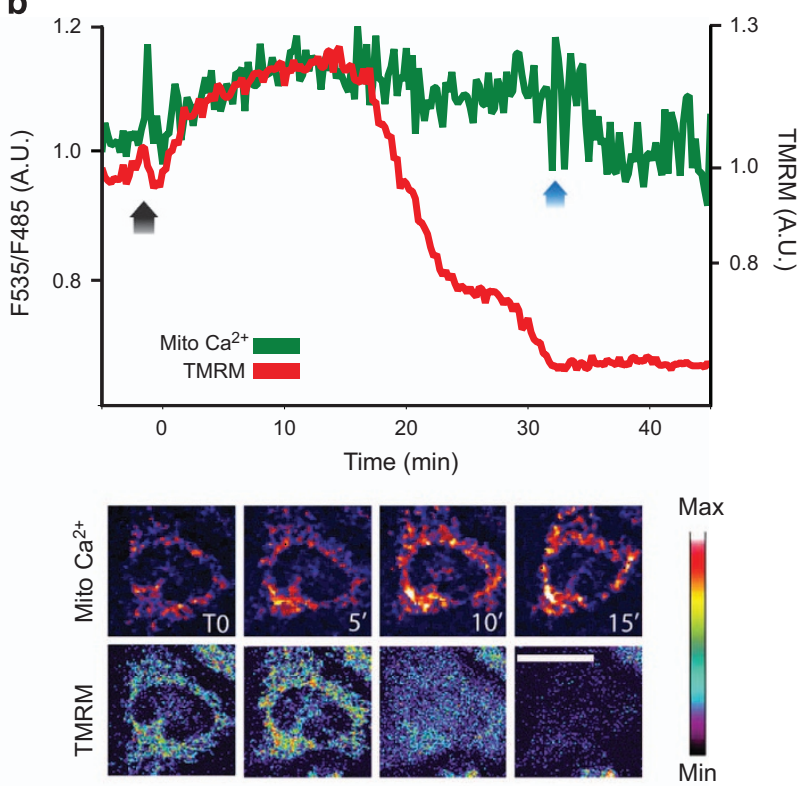

Max

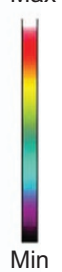

Time after PSY treatment

Figure 3 PSY induces mitochondrial $\mathrm{Ca}^{2+}$ elevations. Representative traces of Mitochondrial $\mathrm{Ca}^{2+}$ (green lines) and potential (red lines) kinetics upon PSY $10 \mu \mathrm{M}$ administration ( $t=0$; black arrows) ( $n=3,>15$ analyzed cells). Time-lapse images of the MTCD2CPV FRET signal and TMRM fluorescence of representative cells are reported below the traces. (a) Apoptotic $\mathrm{Ca}^{2+}$ dysregulation: PSY induces a first mitochondrial $\mathrm{Ca}^{2+}$ peak ( $t=5-8 \mathrm{~min}$ ), and a second, more protracted, $\mathrm{Ca}^{2+}$ elevation before cell lysis (blue arrow). The mitochondrial potential shows a first hyperpolarization followed by progressive leak until cell lysis. (b) Necrotic $\mathrm{Ca}^{2+}$ dysregulation: only the first $\mathrm{Ca}^{2+}$ peak is detectable, followed by a progressive $\mathrm{Ca}^{2+}$ increase coupled with fast mitochondrial depolarization until cell lysis that, in this case, occurred at $t=30$ min

to avoid cross talking between TMRM and YFP excitation. Experiments confirmed the co-localization of the MTCD2CPV probe with TMRM (Supplementary Figure S4). Next, we performed time-lapse experiments with MTCD2CPVexpressing cells stained with TMRM. TMRM fluorescence loss was used as time reference to compare mitochondrial $\mathrm{Ca}^{2+}$ recordings and the experiments performed with Fluo-3, with the aim to understand whether the cytoplasmic $\mathrm{Ca}^{2+}$ kinetics was correlated to mitochondrial $\mathrm{Ca}^{2+}$ variations. After at least 30 min of baseline recording, we treated cells with PSY $10 \mu \mathrm{M}$. PSY caused a mitochondrial $\mathrm{Ca}^{2+}$ increase that well correlated with the PSY-induced cytoplasmic $\mathrm{Ca}^{2+}$ increase. Indeed, PSY induced a rapid mitochondrial $\mathrm{Ca}^{2+}$ peak $(t \approx 5-8 \mathrm{~min})$, followed by recovery to baseline levels and by a second more protracted $\mathrm{Ca}^{2+}$ elevation. This last event started before the onset of mitochondrial potential decrease, and was maintained until cell death occurred (Figure 3a). Similarly to what observed for cytoplasmic $\mathrm{Ca}^{2+}$ kinetics, in the subset of PSY-treated cells undergoing necrosis, we measured a single mitochondrial $\mathrm{Ca}^{2+}$ increase and fast mitochondrial potential loss, followed by membranes lysis with no blebbing (Figure $3 \mathrm{~b}$ ). Control experiments were performed by treating cells with the same quantity of solvent (DMSO) and the cells failed to show relevant changes in mitochondrial $\mathrm{Ca}^{2+}$ or potential with respect to the baseline, demonstrating the specificity of PSY effects (Supplementary Figure S3b).

PSY induces intra-mitochondrial ROS production. As our experiments showed a clear PSY effect on mitochondrial $\mathrm{Ca}^{2+}$ and because $\mathrm{Ca}^{2+}$ increase inside mitochondria can induce ROS production, we evaluated whether these two events were coupled during PSY-induced cell death. To this end, we treated $\mathrm{MO} .13$ for $24 \mathrm{~h}$ with PSY 3-10 $\mu \mathrm{M}$; after treatments, cells were stained with MitoTracker Red $\mathrm{CM}-\mathrm{H}_{2} \mathrm{XRos}$ and measured by flow cytometry (Figure $4 \mathrm{a}$ ) as described in Materials and Methods.

Figure $4 \mathrm{~b}$ shows that serum deprivation did not induce a significant increase of mitochondrial ROS; conversely, all PSY concentrations induced relevant mitochondrial ROS production, as we hypothesized (Figure 4c). The maximum oxidative stress was measured for PSY $10 \mu \mathrm{M}$, which led to about a three folds of increase with respect to the value of control cells in $0 \%$ fetal bovine serum $(P<0.001)$.

As flow cytometry gives an averaged picture after $24 \mathrm{~h}$ of treatment, time-lapse experiments by confocal microscopy were carried out to characterize the kinetics of this PSYinduced mitochondrial ROS production. MO3.13 cells were stained with Fluo-3 and MitoTracker Red $\mathrm{CM}-\mathrm{H}_{2}$ XRos to monitor both cytoplasmic calcium and ROS production. PSY induced an early peak in $\mathrm{CM}-\mathrm{H}_{2}$ XRos fluorescence soon after administration; this peak corresponded with the early calcium peak detected by Fluo-3 fluorescence. After this peak, the signal gradually increased reaching its maximum before a second cytoplasmic $\mathrm{Ca}^{2+}$ increase and indicating robust $\mathrm{ROS}$ elevation for $50 \mathrm{~min}<t<200 \mathrm{~min}$ (Figure 4d). This result confirms data obtained by flow cytometry. Moreover, we notice that this cytoplasmic $\mathrm{Ca}^{2+}$ dynamics was qualitatively the same as that reported in the previous section and that $\mathrm{CM}-\mathrm{H}_{2} \mathrm{XRos}$ fluorescence was closely correlated with cytosolic $\mathrm{Ca}^{2+}$ elevations. Control experiments were performed by treating cells with the same quantity of solvent (DMSO) and the cells failed to show any important change in $\mathrm{CM}-\mathrm{H}_{2} \mathrm{XRos}$ 

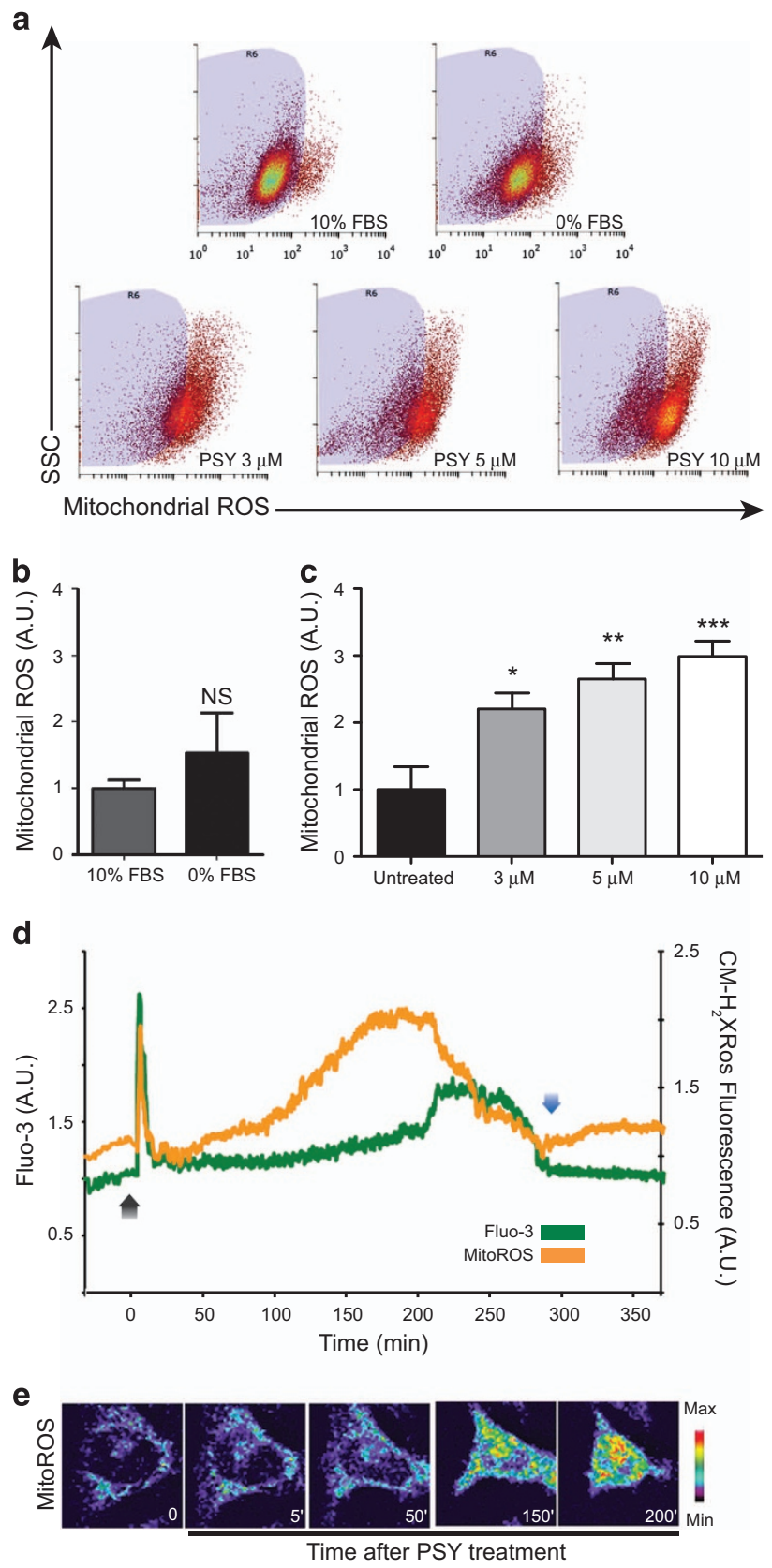

Figure 4 PSY induces mitochondrial ROS production. (a) Representative dotplots of MO3.13 cells stained with Mitotracker Red CM- $\mathrm{H}_{2}$ XRos and analyzed by flow cytometry. The side-scattered light signal (SSC) is also reported. The $\mathrm{R} 6$ region was set by the dot-plot obtained for the control population in (no PSY, 10\% FBS). (b) Mitochondrial ROS production in case of cell starvation ( $0 \% \mathrm{FBS}$ ) and culture maintenance condition (10\% FBS). ( $n=6, t$-test). (c) PSY dose-response quantification of mitochondrial ROS production ( $n=6$, one-way ANOVA (Dunnett's post-hoc test ) PSY 3-10 $\mu \mathrm{M}$ versus Untreated). (d) Representative traces of mitochondrial ROS (orange line) and cytoplasmic $\mathrm{Ca}^{2+}$ (green line) kinetics upon PSY $10 \mu \mathrm{M}$ administration ( $t=0$; black arrow) during apoptotic cell death $(n=3$, $>15$ analyzed cells). PSY induced a first, rapid ROS production peak correlated with the first $\mathrm{Ca}^{2+}$ peak; a second, more protracted, mitochondrial ROS elevation was recorded before cell lysis (blue arrow). (e) Time-lapse images of the Mitotracker Red $\mathrm{CM}-\mathrm{H}_{2} \mathrm{XRos}$ fluorescence of a representative cell is reported below the traces fluorescence or mitochondrial potential with respect to the baseline, demonstrating the specificity of PSY effects (Supplementary Figure S3c).

Reducing extracellular $\mathrm{Ca}^{2+}$ influx extends cell survival and reduces mitochondrial ROS production. Following the observation that PSY treatment triggers calcium elevations, we wondered whether $\mathrm{Ca}^{2+}$ influx from the extracellular environment is causally involved in PSY-induced cell death.

In order to reduce calcium influx from the medium, extracellular $\mathrm{Ca}^{2+}$ content was reduced by using a chelating agent ethylenediaminetetraacetic acid (EDTA). Initially, we performed preliminary dose-response experiments to determine the maximal EDTA concentration not affecting cell viability or ROS production. On the basis of the data reported in Supplementary Figures S5 and S7, we chose $1 \mathrm{mM}$ as the concentration for experiments in the presence of PSY, corresponding to $62 \% \mathrm{Ca}^{2+}$ reduction in our cell-culture medium (see Materials and Methods for details). MO3.13 cells were treated with PSY $3-10 \mu \mathrm{M}$ in presence of EDTA for $24 \mathrm{~h}$, then flow cytometry was carried out to assess viability as previously described (see 'PSY induces apoptotic and necrotic cell death' and Materials and Methods). Our data demonstrate that EDTA can protect MO3.13 from PSYinduced cell death. Indeed, EDTA increased cell viability 1.5fold in case of PSY $5 \mu \mathrm{M}$, and twofold in case of PSY $10 \mu \mathrm{M}$ with respect to cells treated with same concentrations of PSY but without EDTA (Figures $5 \mathrm{a}$ and $\mathrm{b}$ ).

Given this protective effect, we checked whether mitochondrial ROS production was affected by the reduction of calcium influx. Parallel experiments were carried out treating the cells with PSY $3-10 \mu \mathrm{M}$ in the presence of EDTA for $24 \mathrm{~h}$, and then staining with MitoTracker ROS $\mathrm{CM}-\mathrm{H}_{2}$ XRos for flow cytometry analysis. Experiments showed that EDTA could significantly reduce mitochondrial ROS production for all the tested PSY concentrations, demonstrating that nearly the $50 \%$ of mitochondrial ROS production can be prevented by simply reducing extracellular $\mathrm{Ca}^{2+}$ concentration (Figures $5 \mathrm{c}$ and d).

Pharmacological reduction of calcium influx and ROS production by EDTA and N-acetyl-cysteine (NAC) had no synergistic effects on cell survival. Finally, as our data showed that EDTA protection led also to the reduction of mitochondrial ROS, we tested whether a treatment reducing both calcium influx and mitochondrial ROS could produce synergistic effects on cell survival after PSY administration.

In order to test this hypothesis, MO3.13 cells were treated with PSY $10 \mu \mathrm{M}$ for $24 \mathrm{~h}$ in the presence of the antioxidant NAC $5 \mathrm{mM}$. This concentration was chosen according to previous results present in the literature. ${ }^{10}$ Cells were harvested and stained with Mito tracker ROS CM-H2XRos and then analyzed by flow cytometry (Figures $6 a$ and S7). As expected, NAC reduced mitochondrial ROS production; the Mito tracker ROS fluorescence was almost halved with respect to all the samples with same concentrations of PSY but without NAC (Supplementary Figure S6a,b). NAC treatment also significantly inhibited PSY-induced $\mathrm{MO} 3.13$ cell death as revealed by the Annexin V/PI experiments; differences were statistically significant for PSY $10 \mu \mathrm{M}$ (Supplementary Figure S6c,d). However, when we measured the viability of MO3.13 cells 


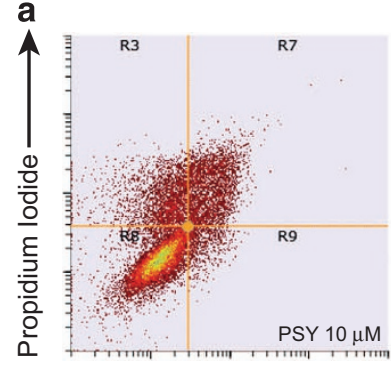

Annexin V

\section{b}
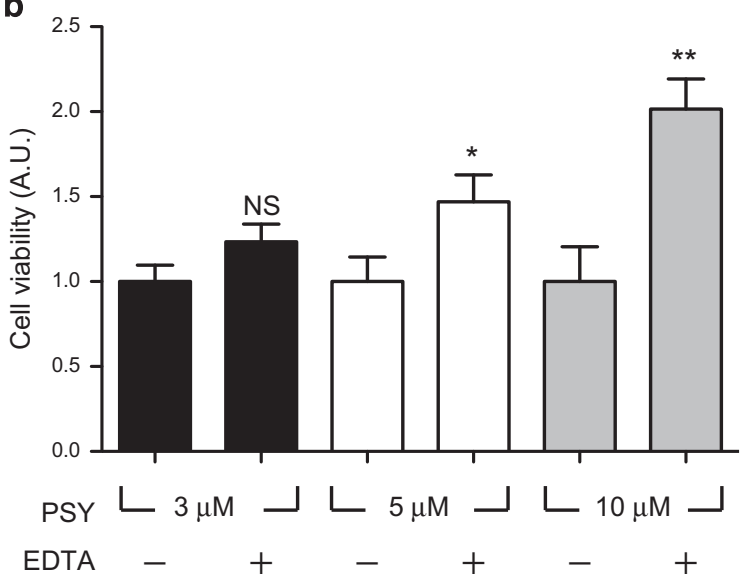

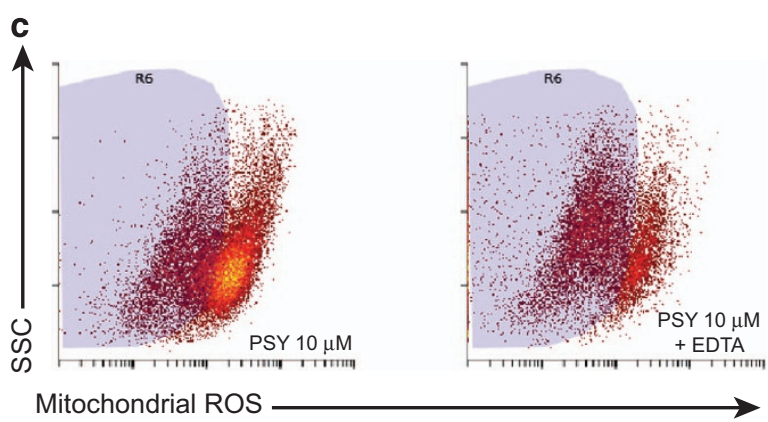

d

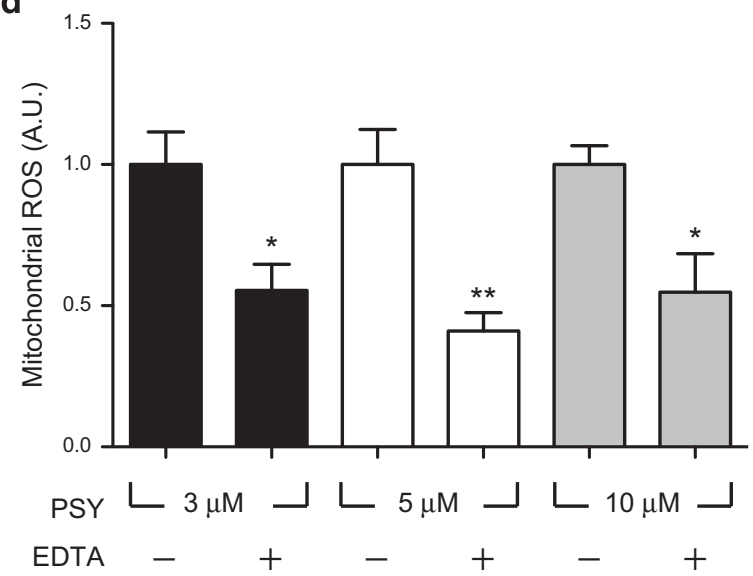

Figure 5 EDTA treatment extends cell survival and reduces mitochondrial ROS production. (a) Representative dot-plots of MO3.13 cells treated with PSY $10 \mu \mathrm{M}$ or with PSY $10 \mu \mathrm{M}$ in presence of EDTA $1 \mathrm{mM}$, stained with Annexin V/propidium iodide and analyzed by flow cytometry: R8, R9, R7 and R3 quadrants define the healthy, apoptotic, secondary necrotic and necrotic populations, respectively. (b) Cell viability quantification of the flow cytometry dot-plots for cells treated with PSY 3-10 $\mu \mathrm{M}$, with $(+)$ of without $(-)$ EDTA $1 \mathrm{mM}$ administration ( $n=7$, t-test EDTA ( - ) versus EDTA (+) for same PSY concentrations). (c) Representative dot-plots of MO3.13 cells treated with PSY $10 \mu$ M or with PSY $10 \mu \mathrm{M}$ in presence of EDTA $1 \mathrm{mM}$, stained with Mitotracker Red CM- $\mathrm{H}_{2} \mathrm{XRos}$ and analyzed by flow cytometry. The side-scattered light signal (SSC) is also reported. The R6 region was set by the dot-plot obtained for the control population in (no PSY, 10\% FBS). (d) Mitochondrial ROS production quantification of the flow-cytometry dot-plots for cells treated with PSY 3-10 $\mu \mathrm{M}$, with (+) of without ( - ) EDTA $1 \mathrm{mM}$ administration $(n=4$, $t$-test EDTA $(-)$ versus EDTA (+) for same PSY concentrations)

treated with PSY for $24 \mathrm{~h}$ in the presence of both EDTA (1 mM) and NAC (5 mM), we did not observe any significant difference with respect to cells treated with EDTA or NAC alone, indicating that these drugs had no synergistic effect on cell survival after PSY treatment (Figures $6 b$ and S7).

\section{Discussion}

GLD is a neurodegenerative disease characterized by the intracellular accumulation of PSY, a cytotoxic sphingolipid. It has been hypothesized that PSY has a crucial role in dysfunction of myelinating cells and white matter loss, but little is known about its specific intracellular effects. The aim of this work is to shed light on specific PSY-induced intracellular pathways that are currently incompletely understood, and that could be impacted by pharmacological treatments with the aim to possibly delay GLD onset and/or slow down its progression.

It is well known that several sphingolipid messengers such as ceramide, glucosyl-ceramide, ceramide-1-P, sphingosine and sphingosine-1-phosphate can regulate intracellular $\mathrm{Ca}^{2+}$ homeostasis. ${ }^{22-26}$ Likewise, few studies in the 90s showed that PSY can mobilize nuclear and cytoplasmic $\mathrm{Ca}^{2+}$ in isolated rat liver nuclei ${ }^{27}$ and in several cultured cell lines (e.g., MC3T3-E1 pre-osteoblasts, ${ }^{28}$ Jurkat T-cells, RINm5F Insulinoma cells ${ }^{29}$ ), respectively. More recently, two papers by
Lloyd-Evans et al. ${ }^{30,31}$ reported $\mathrm{Ca}^{2+}$ release in rat brain microsomes upon PSY treatment, with PSY acting as an agonist of ryanodine receptors. Interestingly, these references documented relevant $\mathrm{Ca}^{2+}$ alterations (as increase in cell cytoplasm and insolated nuclei, and release form microsomes) within $\approx 10$ min from PSY (up to $30 \mu \mathrm{M}$ ) administration, but $\mathrm{Ca}^{2+}$ kinetics on longer time-scales was not investigated. Moreover, in contrast with other lysosomal storage disorders, no clear evidence has been still reported that $\mathrm{Ca}^{2+}$ dysregulation is a pathogenic factor in GLD. ${ }^{32}$ Our data are full in agreement with the above-presented literature, extending the characterization of $\mathrm{Ca}^{2+}$ dynamics until PSY-induced cell death occurs; PSY accumulation is indeed believed to be the main cause of oligodendrocyte loss in GLD. Moreover, in order to make our analysis more relevant for shedding light on GLD pathogenesis, we used a differentiated human oligodendrocyte cell line (starved MO3.13 cells) for all the experiments. MO3.13 cells are indeed widely used for GLD research (see, e.g., Won et al. $^{33}$ and Giri et al. ${ }^{8}$ ) because of their very active sphingolipid metabolism similar to that of primary oligodendrocytes. ${ }^{34}$ Our experiments of $\mathrm{Ca}^{2+}$ chelation directly support the hypothesis that $\mathrm{Ca}^{2+}$ dysregulation by PSY can trigger oligodendrocyte loss.

By treating MO3.13 cells with exogenous PSY, we observed necrotic and apoptotic cell death, as expected. 

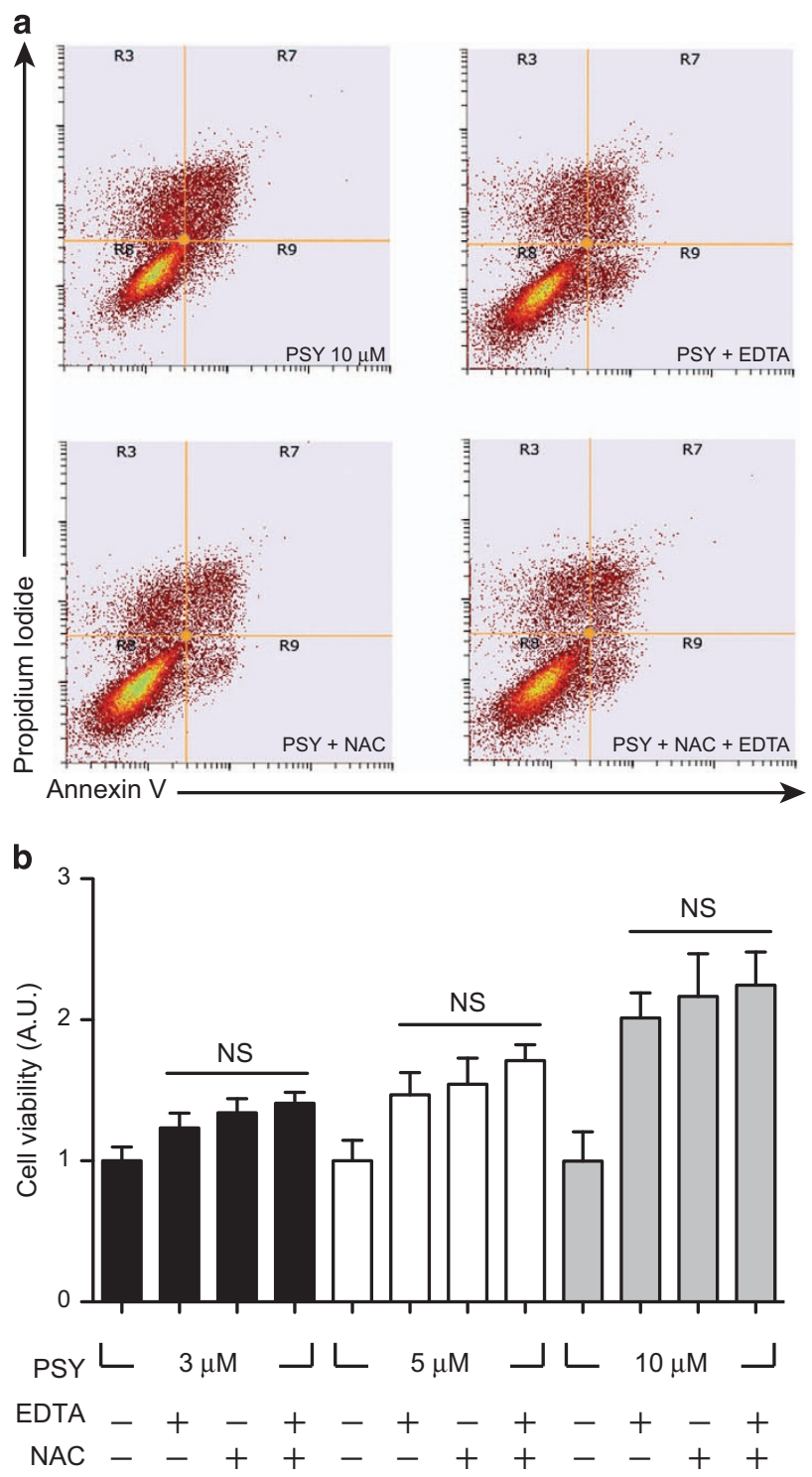

Figure 6 Pharmacological reduction of calcium influx and ROS production by EDTA and $\mathrm{N}$-acetyl-cysteine has no synergistic effect on cell survival. (a) Representative dot-plots of MO3.13 cells treated with PSY $10 \mu \mathrm{M}$ alone, or in combination with EDTA $1 \mathrm{mM}, \mathrm{NAC} 5 \mathrm{mM}$ and EDTA $1 \mathrm{mM}+\mathrm{NAC} 5 \mathrm{mM}$, stained with Annexin V/propidium iodide and analyzed by flow cytometry: R8, R9, R7 and R3 quadrants define the healthy, apoptotic, secondary necrotic and necrotic populations, respectively. (b) Cell viability quantification of the flow cytometry dot-plots for cells treated with PSY $3-10 \mu \mathrm{M}$, with $(+)$ of without $(-)$ EDTA $1 \mathrm{mM}$ and NAC $5 \mathrm{mM}$ administration. ( $n=5$, one-way ANOVA, (Tukey post-hot test): EDTA(+)/NAC( - ) versus EDTA $(-) / \mathrm{NAC}(+)$ versus EDTA(+)/NAC(+) for PSY $3 \mu \mathrm{M}$ and PSY $10 \mu \mathrm{M}$; one-way ANOVA, (Dunns post-hoc test) EDTA(+)/NAC( - ) versus EDTA $(-) / \mathrm{NAC}(+)$ versus $\mathrm{EDTA}(+) / \mathrm{NAC}(+)$ for PSY $5 \mu \mathrm{M})$

The necrotic population died by immediate calcium dysregulation (Figure 2b), whereas the apoptotic population died by delayed calcium dysregulation (Figure $2 \mathrm{a}$ ).$^{21}$ In the apoptotic population, after the mentioned early $\mathrm{Ca}^{2+}$ peak occurring few minutes after treatment, the cytoplasmic $\mathrm{Ca}^{2+}$ presented a second robust elevation during the last phases of apoptosis execution (Figure 2a). These sustained unbalances in intracellular $\mathrm{Ca}^{2+}$ might originate from the activation of specific membrane channels ${ }^{35-37}$ or from a direct permeabilization of the plasma membrane owing to the change in its lipid composition. Structural sphingolipids such as galactosilceramide, one of the substrate of the GALC enzyme, are localized in membrane microdomains called rafts. Rafts are membrane regions enriched in sphingolipids and cholesterol: this composition makes raft structures less fluid than the surrounding phospholipids bilayer, and for this reason, they are involved in cell shaping, cytoskeleton anchoring to the plasma membrane, trafficking of proteins and receptors clustering or regulation. ${ }^{38-40}$ It has been recently demonstrated that PSY specifically accumulates in membrane microdomains in the brain of Twitcher mice and GLD patients, disrupting the raft architecture. Sub-cellular raft fractions resulted enriched in cholesterol as well, indicating a change in the overall raft composition during GLD progression. ${ }^{4}$ Rafts are tightly linked to $\mathrm{Ca}^{2+}$ regulation: the activation of storeoperated $\mathrm{Ca}^{2+}$ influx is modulated by lipid rafts, and the transient receptor channels TRPC1 and TRPC6 are involved in raft-mediated $\mathrm{Ca}^{2+}$ influx. ${ }^{41} \mathrm{~A}$ possible scenario is that the PSY-induced raft disruption can also change the overall membrane $\mathrm{Ca}^{2+}$ permeability and/or determine an active $\mathrm{Ca}^{2+}$ influx mediated by channels anchored to lipid rafts.

Our data demonstrate that the cytoplasmic $\mathrm{Ca}^{2+}$ increase induced by PSY is accompanied by an increase of mitochondrial $\mathrm{Ca}^{2+}$. Mitochondria are organelles that can locally sense the cytoplasmic $\mathrm{Ca}^{2+}$ and are localized in specific subcellular zones where their activity is required. In basal conditions, mitochondrial $\mathrm{Ca}^{2+}$ is maintained at low concentrations $(\approx 100 \mathrm{nM})$, but mitochondria can sense cytoplasmic $\mathrm{Ca}^{2+}$ variations and transiently sustain high $\mathrm{Ca}^{2+}$ concentrations (hundreds of $\mu \mathrm{M}),{ }^{42}$ thus contributing to $\mathrm{Ca}^{2+}$ homeostasis in the cell. Slight mitochondrial $\mathrm{Ca}^{2+}$ increases can regulate the mitochondrial activity by activating the Krebs cycle dehydrogenases, by promoting the supply of oxidable substrates and by regulating the activity of ATP synthase. ${ }^{16}$ This is one of the mechanisms used by cells to reduce the cytoplasmic $\mathrm{Ca}^{2+}$, because ATP production can stimulate the sarco endoplasmic calcium ATPase that transfers $\mathrm{Ca}^{2+}$ from the cytoplasm to the endoplasmic reticulum. ${ }^{43}$ We reported a progressive increase of the mitochondrial potential during the first phases following PSY administration, indicating stimulation of mitochondrial activity and suggesting the activation of the endoplasmic reticulum for storing the $\mathrm{Ca}^{2+}$ excess. Conversely, robust mitochondrial $\mathrm{Ca}+$ elevations lead to ROS production that runs out with mitochondrial membranes destruction, release of cytochrome $\mathrm{C}$ and apoptosis induction. ${ }^{16}$ Following the first $\mathrm{Ca}^{2+}$ peak, we indeed measured a second, sustained $\mathrm{Ca}^{2+}$ increase, and enhancement of mitochondrial ROSs (Figure $4 \mathrm{~d}, 50 \mathrm{~min}<t<200 \mathrm{~min}$ ) before cell death. These data fit with the paradigm of cell death mediated by mitochondrial $\mathrm{Ca}^{2}$ ${ }^{+}$increase and ROS production, as previously hypothesized by Formichi and co-workers. ${ }^{7}$ Interestingly, mitochondrial abnormalities, such as loss of mitochondrial $\mathrm{Ca}^{2+}$ buffering and malfunctioning in the lysosomal-mitochondrial axis, have been also reported in other lysosomal storage disorders. ${ }^{44}$

Therefore, given this evidence that PSY can induce a protracted $\mathrm{Ca}^{2+}$ elevation and mitochondria-mediated cell death, we hypothesized that protection from PSY cytotoxicity could result from reducing the extracellular calcium content and mitochondrial oxidative stress, possibly in a synergic way. 
We found that $\mathrm{Ca}^{2+}$ chelation from the extracellular medium by EDTA (1 mM) improves cell survival following PSY administration (Figure $5 \mathrm{~b}$ ) and reduces mitochondrial ROS production by $50 \%$ (Figure $5 \mathrm{~d}$ ). Similarly, halving ROS production by NAC also results in enhancements of cell viability (Supplementary Figure S6). We finally treated cells with both EDTA and NAC, and we obtained no synergic effect on cell viability (Figure 6).

This finding suggests that $\mathrm{Ca}^{2+}$ influx might be the main cause of mitochondrial ROS production, acting upstream of the mitochondrial oxidative stress induction. Yet, we cannot exclude that the concentrations used for EDTA and NAC were high enough to independently saturate cell protection mechanisms, thus hindering the observation of a possible cumulative effect.

The demonstration of the role of calcium in PSY-induced cell death gives some indications on possible therapeutic targets to be tested in GLD models, such as the raft-associated calcium channels (e.g., the TRPC). Moreover, given the important role of mitochondrial ROS production, antioxidants that specifically target mitochondria might be tested. The MitoQ, for example, is a mitochondria-targeted antioxidant designed to accumulate within mitochondria in vivo in order to protect against oxidative damage. This molecule has already undergone clinical trials in humans, showing optimal results in terms of safety for up to 1 year of treatment. ${ }^{45}$

\section{Conclusions}

In this study, we have followed longitudinally calcium dynamics and mitochondrial ROS during PSY-induced oligodendrocyte cell death. We report that PSY treatment causes elevations of cytosolic and mitochondrial $\mathrm{Ca}^{2+}$ that are coupled to mitochondrial ROS production. Chelation of extracellular calcium decreases intra-mitochondrial ROS production and enhances cell viability. Antioxidant treatment also reduces mitochondrial ROS production and cell loss, but this therapy does not synergize with $\mathrm{Ca}^{2+}$ chelation. Altogether, these data provide novel information on the intracellular pathways activated during PSY-induced toxicity.

\footnotetext{
Materials and Methods

Cell culture and treatments. Human oligodendrocyte MO3.13 cells (Tebu Bio, Le-Perray-en-Yvelines, France, Cat. No. CLU301-P) were maintained in DMEM medium supplemented with $2 \mathrm{mM} \mathrm{L-glutamine,} 1 \%$ penicillin/streptomicyn and $10 \%$ heat-inactivated fetal bovine serum (GIBCO-Life Technologies, Carlsbad, $\mathrm{CA}, \mathrm{USA}$ ), at $37^{\circ} \mathrm{C}$ in humidified atmosphere containing $5 \% \mathrm{CO} 2$.

MO3.13 cells were seeded at 25000 cells $/ \mathrm{cm}^{2} ; 24 \mathrm{~h}$ after plating, the medium was removed and cells were washed two times with phosphate-buffered saline (PBS) $1 x$. Then, cells were cultured in serum-free medium (DMEM, supplemented with $2 \mathrm{mM}$ L-glutamine, 1\% penicillin/streptomicyn) or serum-free medium+PSY 1-10 $\mu \mathrm{M}$. For EDTA and NAC experiments, cells were pre-treated for 30 min with EDTA $1 \mathrm{mM}$ or/ and NAC $5 \mathrm{mM}$, then treated with PSY 3-10 $\mu \mathrm{M}$ for $24 \mathrm{~h}$. PSY was diluted in DMSO and prepared as a $10 \mathrm{mM}$ stock solution, EDTA was diluted in water and prepared as $1 \mathrm{M}$ stock solution and NAC was diluted in water as $1 \mathrm{M}$ stock solution; control cultures received the same quantity of vehicle (DMSO or/and water). Given the concentrations of $\mathrm{Ca}^{2+}$ and $\mathrm{Mg}^{2+}$ in the culture medium $(1.8 \mathrm{mM}$ and $0.8 \mathrm{mM}$, respectively, for our DMEM), the binding constants for EDTA with $\mathrm{Ca}^{2+}$ and $\mathrm{Mg}^{2+}$ $\left(\log \mathrm{Ka}=10.65\right.$ for $\mathrm{Ca} 2+$ and 8.79 for $\left.\mathrm{Mg}^{2+}\right)$ and the final concentration od EDTA $(1 \mathrm{mM})$, the theoretical amount of free $\mathrm{Ca}^{2+}$ and free $\mathrm{Mg}^{2+}$ in the medium supplemented with EDTA is calculated to be approximately $0.8 \mathrm{mM}$ for both ions (i.e., in these conditions, essentially no $\mathrm{Mg}^{2+}$ is complexed), that is, $\approx 60 \%$ reduction in $\mathrm{Ca}^{2}$ ${ }^{+}$content. We also measured the pH in DMEM and DMEM+EDTA ( $\left.1 \mathrm{mM}\right)$ and we did not find important medium acidification ( $\mathrm{pH}$ from 7.4 to 7.25 ).
}

Annexin V/PI cell death assay. After treatments, M03.13 cells were harvested and spun for $5 \mathrm{~min}$ at $1400 \mathrm{rpm}$; the obtained pellets were washed with PBS $1 \times$ and spun again for $5 \mathrm{~min}$ at $1400 \mathrm{rpm}$. Cells were re-suspended in binding buffer ( $10 \mathrm{mmol} / \mathrm{l} \mathrm{HEPES}, 135 \mathrm{mmol} / / \mathrm{NaCl}, 5 \mathrm{mmol} / / \mathrm{CaCl}_{2}$ ) containing Annexin V-FITC conjugate $1 \mu \mathrm{M}$ (Invitrogen-Life Technologies, Carlsbad, CA, USA) and PI $100 \mathrm{nM}$ (Sigma-Aldrich, St. Louis, MO, USA) and incubated in ice for $30 \mathrm{~min}$. Flow cytometry was performed by using a S3 flow cytometer (Bio-Rad, Hercules, CA, USA) equipped with 488 and $561 \mathrm{~nm}$ diode-pumped solid-state lasers. Annexin V-FITC was excited using the $488 \mathrm{~nm}$ laser, and fluorescence was collected through a 552/50 nm band-pass filter and $505 \mathrm{~nm}$ long-pass filter; PI was excited with the $561 \mathrm{~nm}$ laser and the fluorescence was collected through a $605 / 40 \mathrm{~nm}$ band-pass filter and a $570 \mathrm{~nm}$ long-pass fiter. At least 20000 gated events were acquired for each sample. Data were analysed using the Bio-Rad ProSort software.

Hoechst staining for condensed nuclei. Cells were fixed with paraformaldeyde $2 \%$ for $30 \mathrm{~min}$ at room temperature; after fixation, cells were washed once with PBS and stained with Hoechst $33342(1 \mu \mathrm{g} / \mathrm{ml}$ in PBS, Invitrogen-Life Technologies) for $30 \mathrm{~min}$. Fluorescence and phase contrast images were acquired by using an Eclipse Ti inverted microscope (Nikon, Tokyo, Japan) equipped with a $\times 20$ air Nikon objective, N.A. 0.75, ApoFluor and a CCD ORCA R2 (Hamamatsu, Shizuoka, Japan). Hoechst 33342 was excited by a mercury arc lamp using a 450/50 nm band-pass filter, and emission was collected using an UV-2E/C filter (Nikon). Cell death was qualitatively assessed on the basis of cellular and nuclear morphology evaluating the presence of condensed or fragmented nuclei.

SDS-PAGE and western blotting. A total of $1 \times 10^{6} \mathrm{MO} .13$ cells were seeded in $10 \mathrm{~cm}$ diameter dishes; $24 \mathrm{~h}$ after plating, the medium was removed, cells were washed two times with PBS and incubated for $24 \mathrm{~h}$ in serum-free medium, or in medium with $10 \%$ fetal bovine serum (control condition for this experiment). Preparation of cell lysates and western blotting were carried out as described in Reimertz et al. ${ }^{46}$ The resulting blots were probed with: (i) a rabbit polyclonal antimyelin based protein antibody (1:500, AbCam, Cambridge, UK, ab62631); (ii) a mouse monoclonal anti-tubulin antibody $(1: 50000$, Sigma-Aldrich). Horseradish peroxidase-conjugated secondary antibodies (1:10000, Pierce, Northumberland, UK) were detected using SuperSignal West Pico Chemiluminescent Substrate (Pierce) and imaged using a ImageQuant LAS-4000 imaging system (GE Healthcare, Little Chalfont, UK).

Immunofluorescence and confocal analysis. MO3.13 cells were plated on glass cover slips $\left(25000 \mathrm{cell} / \mathrm{sm}^{2}\right)$ into 24-well plates for 2 days and then treated with serum-free medium. After $24 \mathrm{~h}$ of treatment, cells were fixed for $30 \mathrm{~min}$ in $2 \%$ paraformaldehyde, permeabilised with $0.1 \%$ Tween-20 and blocked with $5 \%$ horse serum for $15 \mathrm{~min}$. Cells were then incubated overnight with antimyelin basic protein antibody (1:50, AbCam ab62631). Cover slips were washed with PBS (10 min for three times), incubated with anti-rabbit biotinylated secondary antibody (Jackson Immuno Research Europe, Suffolk, UK, 1:500) for $90 \mathrm{~min}$, washed with PBS and then stained with Alexa 488 Streptavidin (Molecular Probes, Netherlands, $1: 1000$ ) for $30 \mathrm{~min}$. Finally, the cover slips were mounted onto glass microscope slides in presence of Vectashield mounting medium with DAPI (Vector Laboratories inc. Burlingame, CA, USA). Confocal images were acquired as z-stacks by using a scanned on $z$ axis with a SP2 TCS-NT Leica (Nussloch, Germany) laser scanning confocal microscope, equipped with $40 \times 1.2$ NA Plan Apo oil objective. The optical slice was set to $1 \mu \mathrm{M}$ (FWHM).

Mitochondrial ROS detection by flow cytometry. MO3.13 cells were seeded in 24 well plates $\left(25000\right.$ cells $\left./ \mathrm{cm}^{2}\right)$ and treated as described before; after treatments, the cells were stained with MitoTracker Red CM- $\mathrm{H}_{2} \mathrm{XRos}$ (Molecular Probes-Life Technologies, Carlsbad, CA, USA) for $30 \mathrm{~min}$ at $37^{\circ} \mathrm{C}$ in humidified

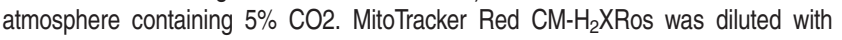
anhydrous DMSO to obtain $1 \mathrm{mM}$ of stock solution and used $1 \mu \mathrm{M}$ as final concentration. After staining, cells were harvested and spun for $5 \mathrm{~min}$ at $1400 \mathrm{rpm}$; the obtained pellets were washed with PBS and spun again for 5 min at $1400 \mathrm{rpm}$. Flow cytometry was performed by using a S3 flow cytometer (Bio-Rad) equipped with a $488 \mathrm{~nm}$ and 561 diode-pumped solid-state lasers. MitoTracker Red CM$\mathrm{H}_{2}$ XRos was excited with a $561 \mathrm{~nm}$ laser and fluorescence emission was collected through a $605 / 40 \mathrm{~nm}$ band-pass filter and $570 \mathrm{~nm}$ long-pass fiter. At least 20000 gated events were acquired for each sample. Data were analyzed using the Bio-Rad ProSort Software. 
Cytoplasmic $\mathrm{Ca}^{2+}$ imaging. MO3.13 cells $\left(50000 \mathrm{cell} / \mathrm{cm}^{2}\right.$ ) were plated in Will Co (Amsterdam, The Netherlands) dishes $24 \mathrm{~h}$ before experiments to reach $50-$ $60 \%$ of confluence and to obtain a stable substrate adhesion. One hour before the experiment, MO3.13 cells were deprived by serum, stained with Fluo-3 AM $1 \mu \mathrm{M}$ for $30 \mathrm{~min}$, and then incubated in serum-free medium with TMRM ( $50 \mathrm{nM})$. TMRM concentration was chosen to avoid quenching of the dye. One hour later, the WillCo dish was mounted into SP2 TCS-NT Leica laser scanning confocal microscope, equipped with $40 \times 1.2$ NA Plan Apo oil objective and with an incubating chamber $\left(\mathrm{T}=37^{\circ} \mathrm{C}\right.$ and $\left.5 \% \mathrm{CO} 2\right)$. Fluo-3 was excited using the 488-nm laser line, and the emitted fluorescence was collected through a 530/30-nm band-pass filter; TMRM was excited using the 568-nm laser line, and the emitted fluorescence was collected through a 590-nm long-pass filter. Excitatory lights were kept to the minimum power to minimize photodamage and photobleaching. The pinhole of the microscope was set to obtain an optical slice of $0.5 \mu \mathrm{m}$ and samples were scanned two times at $512 \times 512$ pixel resolution with a time interval of $15 \mathrm{~s}$. After at least $30 \mathrm{~min}$ of baseline recording, PSY $10 \mu \mathrm{M}$ was added to the medium and cellular response was recorded for $6-8 \mathrm{~h}$.

Transfections. For mitochondrial calcium measurements, $1 \times 10^{5} \mathrm{MO} .13$ cells were plated into $1 \mathrm{~cm}$ diameter WillCo dishes and, $24 \mathrm{~h}$ later, transfected with cDNA encoding for the mitochondrial cameleon FRET probe MTCD2CPV (kindly supplied from Prof. Roger Tsien). ${ }^{47}$ In this construct, a $\mathrm{Ca}^{2+}$-responsive element (calmodulin, $\mathrm{CaM}$ ) is present that, case of $\mathrm{Ca}^{2+}$ variations, alters the efficiency of FRET between two fluorescent proteins. The transfection solution was prepared diluting $1 \mu \mathrm{g}$ of plasmid and $1 \mu \mathrm{l}$ of Lipofectamine 2000 (Life Technologies, Carlsbad, CA, USA) reagent in OPTIMEM medium (Life Technologies) following the manufacturer's instructions, and incubated at room temperature for $30 \mathrm{~min}$. After liposome reaction, solutions were used to transfect the cells. After 90 min incubation, the transfection medium was removed and replaced with fresh medium. Experiments were performed $72 \mathrm{~h}$ after the transfection to have $\approx 80 \%$ of the cells with the correct MTCD2CPV localization into the mitochondria. Ninety minutes before experiments, MO3.13 cells were deprived of serum and after $1 \mathrm{~h}$ stained for $1 \mathrm{~h}$ with TMRM $50 \mathrm{nM}$ and mounted into the incubator chamber for the acquisition in time-lapse at $37^{\circ} \mathrm{C}$ in a humidified atmosphere containing $5 \% \mathrm{CO}$. Images were collected with a time interval of $15 \mathrm{~s}$, with a resolution of $512 \times 512$ pixels. The MTCD2CPV calcium probe was excited with the 458 laser line of a SP2 TCS-NT Leica confocal microscope and the emissions of CFP and YFP were recorded at 485 and $535 \mathrm{~nm}$, respectively. TMRM was excited with 568-nm laser line in sequential acquisition after cameleon recording to exclude YFP excitation and cross-talking. For each time point, both fluorescent and transmitted light images were acquired. The quantification was performed by using the MetaMorph 5.0 software (Universal Imaging, West Chester, PA, USA) expressing the calcium concentration as ratio of emissions FRET/CFP (535/485). The images were elaborated with the plug-in 'FRET analyzer' of the Image $\mathrm{J}$ free software (http://imagej.nih.gov/ij).

$\mathrm{Ca}^{2+}$ and mitochondrial ROS evaluation. MO3.13 cells (50000 cells/ $\mathrm{cm}^{2}$ ) were plated into WillCo dishes $24 \mathrm{~h}$ before experiments to reach $50-60 \%$ of confluence and to obtain a stable substrate adhesion. After Fluo-3 AM loading (as described in 'Cytoplasmic $\mathrm{Ca}^{2+}$ imaging'), medium was removed and replaced with serum-free medium with MitoTracker Red CM- $\mathrm{H}_{2} \mathrm{XRos} 1 \mu \mathrm{M}$. Cells were stained for $30 \mathrm{~min}$ at $37^{\circ} \mathrm{C}$ in a humidified atmosphere containing $5 \% \mathrm{CO} 2$ and then imaged by using a SP2 TCS-NT Leica confocal microscope, equipped with $40 \times 1.2$ NA Plan Apo oil objective and with an incubating chamber $\left(T=37^{\circ} \mathrm{C}, 5 \% \mathrm{CO}\right)$. Fluo-3 signal was recorded as described in 'Cytoplasmic $\mathrm{Ca}^{2+}$ imaging'. Mito Tracker Red $\mathrm{CMH}_{2}$ XRos was excited using the 568-nm laser line, and the emitted fluorescence was collected through a 590-nm long-pass filter. Excitatory lights were kept to the minimum power to minimize photodamage and photobleaching. The pinhole of the microscope was set to obtain an optical slice of $0.5 \mu \mathrm{m}$ and samples were scanned two times at $512 \times 512$ pixel resolution with a time interval of $15 \mathrm{~s}$. After at least of 30 min of baseline recording, PSY was added to the medium and cells responses was recorded for $6-8 \mathrm{~h}$.

Statistical analysis. Inferential statistics was used to compare datasets from different experimental groups. All the experiments were repeated at least three times independently (in figure legends, ' $r$ ' indicates the number of the performed experiments). Data are reported as the averaged value of the means of single experiments $+/-$ the standard error of the mean (mean $+/-$ S.E.M.). Each distribution of the means or, when required, of full datasets has been tested for normality (e.g., by the Shapiro-Wilk normality test). For parametric data, Student's t-test (unpaired, two-tailed) or one-way ANOVA (Tukey's or Dunnett's post tests) were used; for non-parametric data, Mann-Whitney test (two-tailed) or KruskalWallis test was used. Minimum statistical significance will be set for $P$ values $<0.05$ $\left({ }^{\star} P<0.05 ;{ }^{\star \star} P<0.01 ;{ }^{* \star} P<0.005\right)$. NS stands for 'not significant'.

\section{Conflict of Interest}

The authors declare no conflict of interest.

Acknowledgements. This work was supported in part by Telethon grant GGP11116 to Dr. Matteo Caleo. The authors thank Dr. Mirella Filocamo (Gaslini Hospital, Genova (Italy)) and Dr. Mercedes Garcia Gil (University of Pisa) for fruitful discussions, Prof Roger Tsien and Dr. Laura Colombaioni for the supply of the mitochondrial $\mathrm{Ca}^{2+}$ probe.

1. Wenger $\mathrm{D}$ a, Rafi $\mathrm{M}$ a, Luzi $\mathrm{P}$. Molecular genetics of Krabbe disease (globoid cell leukodystrophy): diagnostic and clinical implications. Hum Mutat 1997; 10: 268-279.

2. Suzuki K. Twenty five years of the "psychosine hypothesis": a personal perspective of its history and present status. Neurochem Res 1998; 23: 251-259.

3. White AB, Givogri Ml, Lopez-Rosas A, Cao H, van Breemen R, Thinakaran G et al. Psychosine accumulates in membrane microdomains in the brain of krabbe patients, disrupting the raft architecture. J Neurosci 2009; 29: 6068-6077.

4. Hawkins-Salsbury JA, Parameswar AR, Jiang X, Schlesinger PH, Bongarzone E, Ory DS et al. Psychosine, the cytotoxic sphingolipid that accumulates in globoid cell leukodystrophy, alters membrane architecture. J Lipid Res 2013; 54: 3303-3311.

5. Teixeira CA, Miranda CO, Sousa VF, Santos TE, Malheiro AR, Solomon M et al. Early axonal loss accompanied by impaired endocytosis, abnormal axonal transport, and decreased microtubule stability occur in the model of Krabbe's disease. Neurobiol Dis 2014; 66: 92-103.

6. Pannuzzo G, Cardile V, Costantino-Ceccarini E, Alvares E, Mazzone D, Perciavalle V. A galactose-free diet enriched in soy isoflavones and antioxidants results in delayed onset of symptoms of Krabbe disease in twitcher mice. Mol Genet Metab 2010; 100: 234-240.

7. Formichi P, Radi E, Battisti C, Pasqui A, Pompella G, Lazzerini PE et al. Psychosine-induced apoptosis and cytokine activation in immune peripheral cells of Krabbe patients.J Cell Physiol 2007; 212: 737-743.

8. Giri S, Khan M, Rattan R, Singh I, Singh a K. Krabbe disease: psychosine-mediated activation of phospholipase A2 in oligodendrocyte cell death. J Lipid Res 2006; 47: 1478-1492.

9. Haq E, Giri S, Singh I, Singh AK. Molecular mechanism of psychosine-induced cell death in human oligodendrocyte cell line. J Neurochem 2003; 86: 1428-1440.

10. Khan M, Haq E, Giri S, Singh I, Singh AK. Peroxisomal participation in psychosine-mediated toxicity: implications for Krabbe's disease. J Neurosci Res 2005; 80: 845-854.

11. Haq E, Contreras M a, Giri S, Singh I, Singh AK. Dysfunction of peroxisomes in twitcher mice brain: a possible mechanism of psychosine-induced disease. Biochem Biophys Res Commun 2006; 343: 229-238.

12. Orrenius S, Nicotera P. The calcium ion and cell death. J Neural Transm Suppl 1994; 43: 1-11.

13. Bootman MD, Collins TJ, Peppiatt CM, Prothero LS, MacKenzie L, De Smet $P$ et al. Calcium signalling-an overview. Semin Cell Dev Biol 2001; 12: 3-10.

14. Pizzo P, Drago I, Filadi R, Pozzan T. Mitochondrial Ca2+ homeostasis: mechanism, role, and tissue specificities. Pflugers Arch 2012; 464: 3-17.

15. Denton RM, McCormack JG. On the role of the calcium transport cycle in heart and other mammalian mitochondria. FEBS Lett 1980; 119: 1-8.

16. Duchen MR. Mitochondria, calcium-dependent neuronal death and neurodegenerative disease. Pflugers Arch 2012; 464: 111-121.

17. Mcconkey DJ, Orrenius S. Signal transduction pathways in apoptosis. Stem Cells 1996; 146: 619-631.

18. Tirodkar TS, Voelkel-Johnson C. Sphingolipids in apoptosis. Exp Oncol 2012; 34: 231-242.

19. Obinata H, Hla T. Assessment of sphingosine-1-phosphate activity in biological samples by receptor internalization and adherens junction formation. Methods Mol Biol 2012; 874: 69-76.

20. Scaduto RC, Grotyohann LW. Measurement of mitochondrial membrane potential using fluorescent rhodamine derivatives. Biophys J 1999; 76(1 Pt 1): 469-477.

21. D'Orsi B, Bonner H, Tuffy LP, Düssmann H, Woods I, Courtney MJ et al. Calpains are downstream effectors of bax-dependent excitotoxic apoptosis. J Neurosci 2012; 32: 1847-1858.

22. McConkey DJ, Orrenius S. The role of calcium in the regulation of apoptosis. J Leukoc Biol 1996; 59: 775-783.

23. Lucki NC, Sewer MB. Nuclear sphingolipid metabolism. Annu Rev Physiol 2012; 74: 131-151.

24. Hinkovska-Galcheva V, Shayman JA. Ceramide-1-phosphate in phagocytosis and calcium homeostasis. Adv Exp Med Biol 2010; 688: 131-140. 
25. Spiegel S, Merrill AH Jr. Sphingolipid metabolism and cell growth regulation. FASEB J 1996; 10: $1388-1397$.

26. Stenovec M, Trkov S, Kreft M, Zorec R. Alterations of calcium homoeostasis in cultured rat astrocytes evoked by bioactive sphingolipids. Acta Physiol (Oxf) 2014; 212: 49-61.

27. Catalán RE, Miguel BG, Calcerrada MC, Ruiz S, Martínez a M. Sphingolipids increase calcium concentration in isolated rat liver nuclei. Biochem Biophys Res Commun 1997; 238: 347-350.

28. Liu R, Farach-Carson MC, Karin NJ. Effects of sphingosine derivatives on MC3T3-E1 pre-osteoblasts: psychosine elicits release of calcium from intracellualr stores. Biochem Biophys Res Commun 1995; 214: 676-684.

29. Himmel HM, Meyer zu Heringdorf D, Windorfer B, van Koppen CJ, Ravens U, Jakobs KH. Guanine nucleotide-sensitive inhibition of L-type $\mathrm{Ca} 2+$ current by lysosphingolipids in RINm5F insulinoma cells. Mol Pharmacol 1998; 53: 862-869.

30. Lloyd-Evans E, Pelled D, Riebeling C, Futerman AH. Lloyd-evans Lyso-glycosphingolipids mobilize calcium from brain microsomes via multiple mechanisms. Biochem J 2003; 375(Pt 3): 561-565.

31. Lloyd-Evans E, Pelled D, Riebeling C, Bodennec J, de-Morgan A, Waller H, Schiffmann R, Futerman $\mathrm{AH}$. Glucosylceramide and glucosylsphingosine modulate calcium mobilization from brain microsomes via different mechanisms. J Biol Chem 2003; 278: 23594-23599.

32. Vitner EB, Platt FM, Futerman AH. Common and uncommon pathogenic cascades in lysosomal storage diseases. J Biol Chem 2010; 285: 20423-20427.

33. Won JS, Kim J, Paintlia MK, Singh I, Singh AK. Role of endogenous psychosine accumulation in oligodendrocyte differentiation and survival: implication for Krabbe disease. Brain Res 2013; 1508: 44-52.

34. Buntinx M, Vanderlocht J, Hellings N, Vandenabeele F, Lambrichts I, Raus $\mathrm{J}$ et al. Characterization of three human oligodendroglial cell lines as a model to study oligodendrocyte injury: morphology and oligodendrocyte-specific gene expression. J Neurocytol 2003; 32 : 25-38.

35. Pietrobon D, Di Virgilio F, Pozzan T. Structural and functional aspects of calcium homeostasis in eukaryotic cells. Eur J Biochem 1990; 193: 599-622.

36. Berridge MJ. Capacitative calcium entry. Biochem J 1995; 312(Pt 1): 1-11.

37. Berridge MJ. Elementary and global aspects of calcium signalling. J Exp Biol 1997; 200(Pt 2): 315-319.

38. Simons K, Sampaio JL. Membrane organization and lipid rafts. Cold Spring Harb Perspect Biol 2011; 3: a004697.
39. Chidlow Jr JH, Sessa WC. Caveolae, caveolins, and cavins: complex control of cellular signalling and inflammation. Cardiovasc Res 2010; 86: 219-225.

40. Hanzal-Bayer MF, Hancock JF. Lipid rafts and membrane traffic. FEBS Lett 2007; 581 2098-2104.

41. Galan C, Woodard GE, Dionisio N, Salido GM, Rosado JA. Lipid rafts modulate the activation but not the maintenance of store-operated $\mathrm{Ca}(2+)$ entry. Biochim Biophys Acta 2010; 1803: 1083-1093.

42. Spät A, Szanda G, Csordás G, Hajnóczky G. High- and low-calcium-dependent mechanisms of mitochondrial calcium signalling. Cell Calcium 2008; 44: 51-63.

43. Wuytack F, Raeymaekers L, Missiaen L. Molecular physiology of the SERCA and SPCA pumps. Cell Calcium 2002; 32: 279-305.

44. Kiselyov K, Muallem S. Mitochondrial Ca2+ homeostasis in lysosomal storage diseases. Cell Calcium 2008; 44: 103-111.

45. Smith RA, Murphy MP. Animal and human studies with the mitochondria-targeted antioxidant MitoQ. Ann N Y Acad Sci 1201, 2010: 96-103.

46. Reimertz C, Kögel D, Lankiewicz S, Poppe M, Prehn JH. Ca(2+)-induced inhibition of apoptosis in human SH-SY5Y neuroblastoma cells: degradation of apoptotic protease activating factor-1 (APAF-1). J Neurochem 2001; 78: 1256-1266.

47. Palmer AE, Giacomello M, Kortemme T, Hires SA, Lev-Ram V, Baker D et al. Ca2+ indicators based on computationally redesigned calmodulin-peptide pairs. Chem Biol 2006; 13: $521-530$.

Cell Death and Disease is an open-access journal published by Nature Publishing Group. This work is licensed under a Creative Commons Attribution 4.0 International Licence. The images or other third party material in this article are included in the article's Creative Commons licence, unless indicated otherwise in the credit line; if the material is not included under the Creative Commons licence, users will need to obtain permission from the licence holder to reproduce the material. To view a copy of this licence, visit http://creativecommons.org/licenses/by/4.0

Supplementary Information accompanies this paper on Cell Death and Disease website (http://www.nature.com/cddis) 